\title{
Magnetic Ordering of Dangling Bond Networks on Hydrogen-Deposited Si(111) Surfaces
}

\author{
Susumu Okada, ${ }^{1,2}$ Kenji Shiraishi, ${ }^{1,2}$ and Atsushi Oshiyama ${ }^{1,2}$ \\ ${ }^{1}$ Institute of Physics and Center for Computational Physics, University of Tsukuba, 1-1-1 Tennodai, Tsukuba 305-8571, Japan \\ ${ }^{2}$ Research Consortium for Synthetic Nano-Function Materials Project (SYNAF), \\ National Institute of Advanced Industrial Science and Technology (AIST), 1-1-1 Umezono, Tsukuba 305-8568, Japan
} (Received 26 April 2002; published 17 January 2003)

\begin{abstract}
Based on total-energy electronic-structure calculations within the density-functional theory, we find that a high spin state is realized for an ultimate dangling bond unit on an otherwise hydrogen-covered $\mathrm{Si}(111)$ surface. We further propose a systematic method of constructing nanometer-scale dangling bond networks that exhibit the ferrimagnetic spin ordering. The interplay between the electron-electron interaction and the surface reconstruction is elucidated.
\end{abstract}

DOI: 10.1103/PhysRevLett.90.026803

Miniaturization in semiconductor technology is urging us to understand and predict microscopic atomic processes on surfaces and at interfaces of materials, and then to clarify their possible applications in the technology. Recently, the scanning probe technique has emerged as a promising tool to manipulate atoms on semiconductor surfaces [1-4]. It was indeed demonstrated that $\mathrm{H}$ atoms on the $\mathrm{H}$-covered $\mathrm{Si}(001)$ surfaces are removed along a Si dimer row in a controlled way [2-4], exposing a dangling bond (DB) array on the surfaces. In this Letter, we theoretically explore the possibility of magnetic ordering in nanometer-scale networks of DBs on Si.

Dangling bonds generated at surfaces of covalent materials are well known to play crucial roles in atomic structures of the surfaces: Reconstruction of surface atoms usually reduces DB energies, albeit an increase in stress energies. Such competition between electronic and elastic energies produces a rich variety of surface reconstructions [5]. As for $\mathrm{Si}(111)$ surfaces, the top-layer $\mathrm{Si}$ atoms constitute a triangular lattice and the buckling of the atoms along the [110] direction leads to a $2 \times 1$ structure [6]. Yet the observed $2 \times 1$ is now confirmed to have a more complicated $\pi$-bonded chain structure in which five-member and seven-member rings appear alternatively [7]. The $7 \times 7$ structure is also explained by a beautiful combination of dimers, adatoms, and stacking faults (DAS model) [8]. Hydrogen adsorption generally terminates DBs and then modifies the balance in energetics: A low-energy electron diffraction pattern reveals that the $2 \times 1$ structure changes to the $1 \times 1$ structure upon $\mathrm{H}$ exposure and that the transition is completed at about 0.5 monolayer $[9,10]$. The surface reconstruction crucially depends on the extent of the $\mathrm{H}$-free region. On the other hand, due to the localized nature of the DBs, the electron-electron interaction is expected to be important for atomic and electronic structures of the surfaces. The local spin density calculation [11] has indeed shown that a spin density wave with the $2 \times 1$ periodicity takes place on the clean $\mathrm{Si}(111)$ as a metastable state. The nanometer-scale DB networks generated on the $\mathrm{Si}(111)$ partially covered by hydrogen are therefore an interesting
PACS numbers: 73.20.-r, 73.22.-f, 75.75.+a

stage where competition between the lattice distortion and the electron interaction is essential in surface properties.

We here find that the magnetic ordering on the hydrogenated $\mathrm{Si}(111)$ surface is realized by forming DB networks in which the surface reconstruction is appropriately suppressed. The density-functional calculations reveal that an ultimate triangle unit consisting of four DBs on an otherwise $\mathrm{H}$-covered $\mathrm{Si}(111)$ surface exhibits a high spin state, $S=1$. We further propose different types of hexagonal networks of the ultimate DB units that clearly exhibit the ferrimagnetic ordering. The magnetic ordering in the DB nanostructures that we find may be utilized in Si based spintronic devices.

All the calculations have been performed on the basis of the density-functional theory $[12,13]$. We use the local spin density approximation (LSDA) [14,15] to express the exchange-correlation energy of interacting electrons. Norm-conserving pseudopotentials [16] with separable forms [17] are adopted to describe the electron-ion interaction. We consider the partial core correction for the $\mathrm{Si}$ atoms in the treatment of the exchange-correlation energy [18]. The valence wave functions are expanded in terms of a plane-wave basis set with the cutoff energy of $10 \mathrm{Ry}$ [19]. The surface is simulated by a repeating slab model in which six Si atomic layers and the $12 \AA$-vacuum region are included. The bottom of the slab has a bulklike structure with each $\mathrm{Si}$ saturated by an $\mathrm{H}$ atom. On the top of the slab, which simulates the real surface, a $\mathrm{H}$ adlayer and DB networks are considered. We use several lateral periodicities depending on what we consider. As for the integration over the surface Brillouin zone, we use the four $k$ points in the case of the $2 \sqrt{3} \times 2 \sqrt{3}$ lateral cell, or the equivalent number of $k$ points in other cases. Geometry optimization has been performed for all the atoms in the slab except for the bottommost $\mathrm{Si}$ and $\mathrm{H}$ atoms. We use the conjugate-gradient minimization scheme both for the electronic-structure calculation and for the geometry optimization [20]. In the optimized geometries, the remaining force acting on the atoms is less than $0.005 \mathrm{Ry} / \AA$. 
We begin with the stability of the $\pi$-bonded chain structure on the $\mathrm{H}$-adsorbed $\mathrm{Si}(111)$. As stated above, the reconstruction pattern is expected to depend on the extent of the $\mathrm{H}$-free region. We thus remove $\mathrm{H}$ atoms attached to the top-layer Si atoms along the [110] direction and prepare strips of the DBs. We have carried out the density-functional calculations and have found that the $\pi$-bonded structure is unstable against the buckling structure when the width of the DB strips are less than three top-layer atoms in the [112] direction. The DAS configuration which is relevant to the $7 \times 7$ structure obviously requires more extended $\mathrm{H}$-free regions. Hence, the important reconstruction pattern in the nanometer-scale DB networks discussed in this Letter is the buckling of surface atoms.

We now examine the importance of spin polarization effects in the DB network. We have indeed found a high spin state in an ultimate triangle unit (Fig. 1) as described below. The triangle unit consists of three edge DB sites (site $A$ ) and a central DB site (site $B$ ) on an otherwise $\mathrm{H}$ covered $\mathrm{Si}(111)$ surface [21]. It generally undergoes a buckling reconstruction. In one buckling pattern shown in Fig. 1(b), the central $B$ site is displaced downwards compared with the edge $A$ sites, whereas in another pattern, shown in Fig. 1(c), the $B$ site is displaced upward. These bucklings reduce the total energy of the triangle unit. Without the spin polarization, the calculated total energies of the buckled structures, Figs. 1(b) and 1(c), are lower than the nonbuckled geometry shown in Fig. 1(a) by 0.67 and $0.80 \mathrm{eV}$, respectively. The amount of the buckling is about $0.4-0.5 \AA$.

The spin-unpolarized states are not the ground state, however. We have found that the spin is polarized in the nonbuckled structure and in one of the buckled structures. (a)

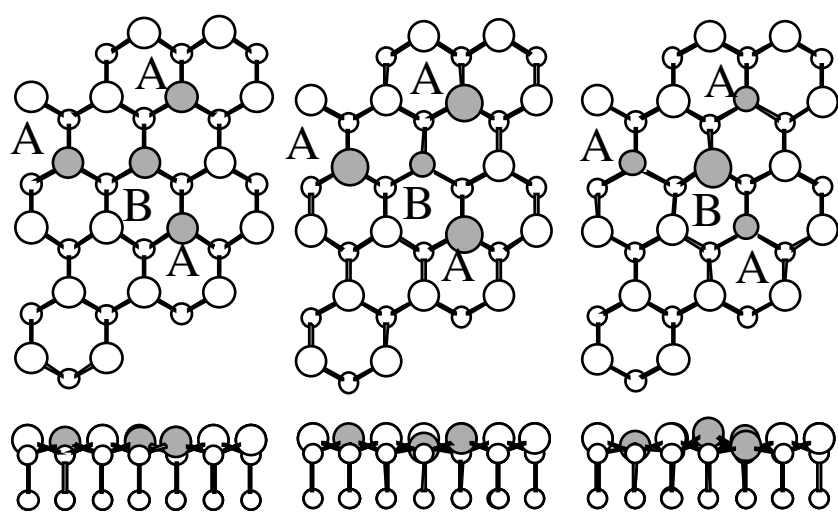

FIG. 1. Top and side views of fully optimized geometries of the ultimate triangle DB unit for the (a) nonbuckled spin-polarized (NP), (b) buckled spin-polarized (BP), and (c) buckled spin-unpolarized (BU) structures. Dark, white, and small white circles denote the $\mathrm{Si}$ with $\mathrm{DB}$, hydrogenated $\mathrm{Si}$, and subsurface $\mathrm{Si}$ atoms, respectively. Only a part of the atoms are shown to avoid visual complexity.
The calculated total energies for the nonbuckled spinpolarized (NP) structure [Fig. 1(a)] and for the buckled spin-polarized (BP) are lower than that for the nonbuckled spin-unpolarized structure by 0.86 and $0.84 \mathrm{eV}$, respectively. The spin density of the most stable NP structure is shown in Fig. 2(a). The majority spin is distributed over the edge $A$ sites, whereas the minority spin is on the central $B$ site. Therefore the calculated spin for the ultimate triangle unit is $S=1$ [22]. The nonbuckled high spin state is energetically the most favorable. The surface reconstruction is not a local atomic displacement of the top-layer atoms but a concerted atomic rearrangement including subsurface atoms. $\mathrm{H}$ atoms covering top-layer $\mathrm{Si}$ atoms around the triangle unit certainly suppress the flexibility for the reconstruction.

Energy bands (Kohn-Sham levels) of the most stable NP structure are shown in Fig. 2(b). There are essentially four surface energy bands originating from four DBs near the Fermi level. Three of the four bunch up and an energy gap appears between the three and the remaining one. The Kohn-Sham orbitals of the three bunched states are distributed over the $A$ DB sites, while the orbital of the remaining state is on the $B$ site. For the majority spin, the bunched states are lower in energy by $0.4 \mathrm{eV}$ than the remaining state, whereas the remaining state is lower for the minority spin by again $0.4 \mathrm{eV}$. Small dispersions of the surface energy bands indicate the localized nature of the DB states and thus possible importance of electron interactions.

It is therefore of interest to map the present system to the Hubbard model. For this purpose, we have carried out

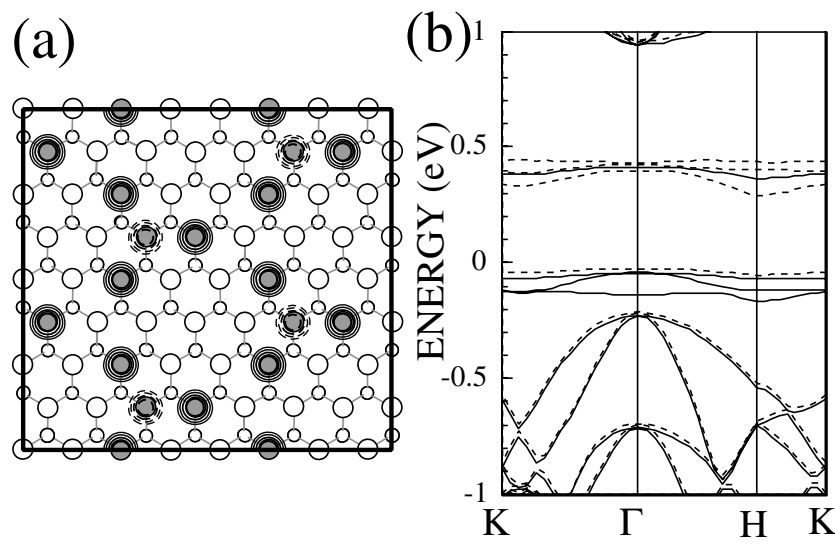

FIG. 2. (a) Contour plot on the (111) plane of the spin density in the NP structure of the triangle DB unit on the Si(111) surface. Positive and negative values of the spin density are shown by solid and dashed lines, respectively. Each contour represents twice (or half) the density of its neighboring contour lines. The lowest contour represents $1.25 \times 10^{-2} e / \AA^{3}$. (b) Energy bands of the NP structure of the DB unit for majority (MJ) and minority (MN) spins along the symmetry lines. The solid and dashed lines denote the bands for the MJ and MN spins, respectively. Electrons are accommodated in the bands with negative energies. 
constrained local spin density approximation calculations for the DB [23] and estimated the value of Coulomb repulsion $U$ in Hubbard Hamiltonian. The obtained value is $U=0.79 \mathrm{eV}$. On the other hand, the electron transfer $t$ is estimated from the bandwidth of the DB state in our local density approximation calculations: i.e., $t=$ $0.080 \mathrm{eV}$. We have further performed the exact diagonalization of Hubbard Hamiltonian [24] for the ultimate triangle DB unit with those $U$ and $t$. The obtained ground state has $S=1$ and the spin configuration is identical to what we have found in LSDA.

The present calculation has unequivocally shown that the high spin state is the ground state for the ultimate triangle DB unit. The DB unit on the $\mathrm{Si}(111)$ surface is thus a promising constituent element for nanometer-scale $\mathrm{Si}$ based spintronic devices. A matrix of the triangle DB units on the $\operatorname{Si}(111)$ surface with an appropriate nanometer-scale read-write device may be the ultimate high density memory unit ( $306.38 \AA$ A $/$ bit), corresponding to a terabite capacity on a typical size of the latest dynamic random access memory chip.

The present finding that the adjacent spins on the triangle DB unit are coupled in an antiparallel way allows us to design a network of DBs on the $\mathrm{Si}(111)$ by removing $\mathrm{H}$ atoms selectively. Our proposal is a hexagonal network of DBs (HDB) on the $\mathrm{Si}(111)$. Figure 3(a) shows its top and side view: $\mathrm{H}$ atoms are removed so that the $\mathrm{DBs}$ are exposed forming the hexagonal network. The network topology is identical to that of a finite-width graphene sheet which has been recently shown to exhibit magnetic

(a)

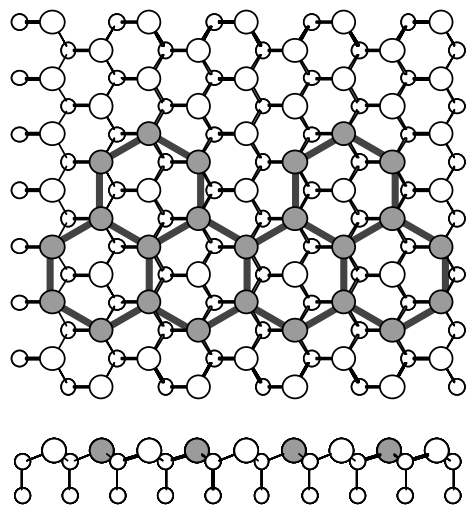

ordering [25]. We have performed geometry optimization for the HDB network [Fig. 3(a)]. Figure 3(b) shows calculated spin density $n_{\uparrow}(\mathbf{r})-n_{\downarrow}(\mathbf{r})$ for the optimized geometry. We have found that the HDB exhibits a ferrimagnetic ordering. The calculated number of polarized spins is one per unit cell. There are two inequivalent sites in the hexagonal cell in Fig. 3(a), and all the DB sites are classified into two sublattices $A$ and $B$ (bipartite lattice). In the HDB structure, the boundary of the DB network in a two-dimensional plane is designed to have an undulating shape so that the number of DBs belonging to each sublattice is different: The number of DBs belonging to the sublattice $A$ is six, while the number belonging to the sublattice $B$ is five $\left(N_{A}=6\right.$ and $\left.N_{B}=5\right)$. It is of interest that the number of the polarized spins in the HDB network equals to $\left|N_{A}-N_{B}\right|$. In all the hexagonal networks of dangling bonds we have examined, it is found that the polarized spins in the network equals to $\mid N_{A}-$ $N_{B} \mid / 2$. We have found that the spin is polarized in one direction at one sublattice and it is in an opposite direction at the other. This finding indicates that a network of DBs with sublattice imbalance $\left(\left|N_{A}-N_{B}\right| \neq 0\right)$ exhibits ferrimagnetic ordering with $S=\frac{1}{2}\left|N_{A}-N_{B}\right|$ in general.

It is important to examine the stability of the magnetic ordering against the structural deformation of the HDB network. In the present case, the DB network has a strip shape where edges are terminated by H-covered $\mathrm{Si}$. The surface flexibility for the reconstruction is larger than in the case of the ultimate triangle DB unit. We have indeed found that the buckling reconstruction takes place in the HDB network: The amount of the buckling is about $0.41 \AA$. Yet even in the buckled HDB network, the spin is polarized and the ferrimagnetic ordering is realized. The total energy of the buckled ferrimagnetic state is almost identical to the nonbuckled ferrimagnetic state. We have thus found an unusual structural and magnetic bistability.

Figure 4(a) shows a more extended hexagonal DB (EHDB) network on the $\mathrm{Si}(111)$. The network is also a bipartite lattice so that the DB sites are classified into either $A$ or $B$ of the two different sublattices. The unit cell contains five DB sites. Three of them, $A_{1}, A_{2}$, and $A_{3}$, belong to the sublattice $A$ and the remaining sites belong to the sublattice $B$ [Fig. 4(a)]. There is thus an imbalance between the two sublattices in the EHDB network. We may thus expect the occurrence of the ferrimagnetic ordering in the EHDB network. At the same time, the extended region of $\mathrm{H}$-free $\mathrm{Si}$ atoms in the EHDB enhances the flexibility for the surface reconstruction, however.

We have performed the total-energy calculations and have found that the most stable state for the EHDB network is the buckled spin-unpolarized (BU) structure. In the BU structure, three DBs belonging to the sublattice $A$ shift downward, whereas the two DBs of the sublattice $B$ shift upward [Fig. 4(a)]. In addition to the above BU structure, we have also found that spin-polarized ferrimagnetic states are realized in both nonbuckled (NP) and 


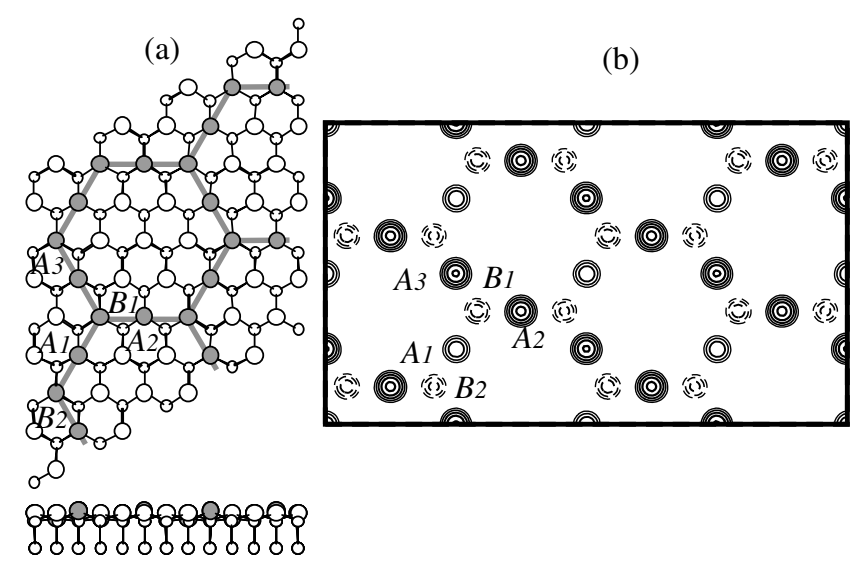

FIG. 4. (a) Top and side views of optimized geometry for the buckled and spin-unpolarized state for the extended hexagonal DB (EHDB) on the Si(111). Dark, white, and small white circles denote the $\mathrm{Si}$ with $\mathrm{DB}$, hydrogenated $\mathrm{Si}$, and subsurface $\mathrm{Si}$ atoms, respectively. Only a part of atoms are shown. The network of the DB is denoted by dashed lines. (b) Contour plot on the (111) plane of spin density $n_{\uparrow}(\mathbf{r})-n_{\downarrow}(\mathbf{r})$ for the nonbuckled spin-polarized state of EHDB network on the $\mathrm{Si}(111)$. Positive and negative values of the spin density are shown by solid and dashed lines, respectively. Each contour represents twice (or half) the density of its neighboring contour lines. The lowest contour represents $1.25 \times 10^{-2} e / \AA^{3}$.

buckled (BP) structures. The calculated total energies are higher by $28 \mathrm{meV}$ per DB for NP and by $14 \mathrm{meV}$ per DB for $\mathrm{BP}$ than that of the BU structure. In the BP structure, three DBs belonging to the sublattice $A$ shift upward, whereas the two DBs of the sublattice $B$ do downward. The calculated spin density $n_{\uparrow}(\mathbf{r})-n_{\downarrow}(\mathbf{r})$ for the NP state is shown in Fig. 4(b). The majority spin is distributed on the sublattice $A$ and the minority on the sublattice $B$.

We have examined several networks of DBs on the $\mathrm{Si}(111)$ surface. The principal finding is that the magnetic ordering is realized for particular DB networks in which the surface flexibility for the reconstruction is sufficiently suppressed. This in turn corroborates a statement that the patterns of the surface reconstruction crucially depend on the exposed area of the top-layer atoms. Also, the present calculation clearly shows that the DB state is substantially localized and that the electron interactions for the DB state are important. This leads to a high spin state for the ultimate triangle DB unit. Hence, the DB networks on the Si surface may be one of the realistic examples of the strongly correlated electron systems. A benefit of this system is the controllability of the ratio $U / t$, guaranteed by the progress in technique of the manipulation of surface atoms.

In summary, we have found that the high spin state is realized in an ultimate triangle dangling bond unit on an otherwise H-covered $\mathrm{Si}(111)$ surface. This opens a possibility of occurrence of magnetic ordering on the premier surface in the current semiconductor technology.

We benefited from conversations with T. Hashizume, K. Okamoto, and H. Tamura. This work was partly sup- ported by ACT-JST (Japan Science and Technology Corporation), Special Research Project on Nanoscience, University of Tsukuba, and NEDO under the Nanotechnology Materials Program and the Collaboration Program. Computations were done at ISSP, University of Tokyo, at SIPC, University of Tsukuba, and at RCCS, Okazaki National Institute.

[1] I.-W. Lyo and Ph. Avouris, Science 253, 173 (1992).

[2] T. C. Shen et al., Science 268, 1590 (1995).

[3] T. Hashizume et al., Jpn. J. Appl. Phys. 35, L1085 (1996).

[4] T. Hitosugi et al., Phys. Rev. Lett. 82, 4034 (1999).

[5] See, for instance, J. J. Boland, Adv. Phys. 42, 129 (1993); K. Oura et al., Surf. Sci. Rep. 35, 1 (1999).

[6] D. Haneman, Phys. Rev. 121, 1093 (1961).

[7] K. C. Pandy, Phys. Rev. Lett. 47, 1913 (1981).

[8] K. Takayanagi et al., J. Vac. Sci. Technol. A 3, 1502 (1985).

[9] H. Ibach and J. E. Rowe, Surf. Sci. 43, 481 (1974).

[10] G. Schulze and M. Henzler, Surf. Sci. 124, 336 (1983).

[11] J. E. Northrup, J. Ihm, and M. L. Cohen, Phys. Rev. Lett. 47, 1910 (1981).

[12] P. Hohenberg and W. Kohn, Phys. Rev. 136, B864 (1964).

[13] W. Kohn and L. J. Sham, Phys. Rev. 140, A1133 (1965).

[14] J. P. Perdew and A. Zunger, Phys. Rev. B 23, 5048 (1981).

[15] D. M. Ceperley and B. J. Alder, Phys. Rev. Lett. 45, 566 (1980).

[16] N. Troullier and J. L. Martins, Phys. Rev. B 43, 1993 (1991).

[17] L. Kleinman and D. M. Bylander, Phys. Rev. Lett. 48, 1425 (1982).

[18] S. G. Louie, S. Froyen, and M. L. Cohen, Phys. Rev. B 26, 1738 (1982).

[19] The cutoff energy of the plane-wave basis set depends on the hardness of the adopted pseudopotentials and are carefully examined by assuring the relevant total-energy differences with accuracy of $0.1 \mathrm{eV}$ : See, e.g., A. Oshiyama, Phys. Rev. Lett. 74, 130 (1995).

[20] O. Sugino and A. Oshiyama, Phys. Rev. Lett. 68, 1858 (1992).

[21] We place the triangle units in $2 \sqrt{3} \times 2 \sqrt{3}$ laterally periodic cells as in Fig. 2(a). Yet the results obtained with this periodic cell are confirmed by using the larger cells which have the periodicities of $4 \sqrt{3} \times 2 \sqrt{3}$ or $3 \sqrt{3} \times 3 \sqrt{3}$.

[22] The higher spin state, e.g., $S=2$, is found to be unstable.

[23] The constrained LSDA calculations have been done, following V. I. Anisimov et al., Phys. Rev. B 48, 16929 (1993): We generate a hole in the valence band top, transfer the electron to the DB, thereby prepare a doubly occupied DB, and perform LSDA calculations with this constraint. The Hubbard $U$ is estimated two ways: One is from the total-energy difference between the constrained and unconstrained electronic configurations, and the other is from the difference in the Kohn-Sham energy level. Both values agree with each other within $10 \mathrm{meV}$.

[24] H. Tamura et al., Phys. Rev. B 65, 085324 (2002).

[25] S. Okada and A. Oshiyama, Phys. Rev. Lett. 87, 146803 (2001). 